\title{
Exportación de tomate en Canarias: ¿un patrón estacional estable?
}

Gloria Martín Rodríguez, Víctor Javier Cano Fernández y José Juan Cáceres Hernández ${ }^{1}$ Dpto. de Economía de las Instituciones, Estadística Económica y Econometría Universidad de La Laguna

RESUMEN: En este trabajo se analiza el patrón estacional de la exportación semanal de tomate canario desde el ingreso de España en la Unión Europea. Un primer examen de los componentes determinísticos de la serie revela la inestabilidad de éstos, claramente vinculada a los cambios en la normativa comercial de acceso a los mercados europeos, así como la presencia de numerosas observaciones anómalas causadas por fenómenos de diversa índole. Estas circunstancias afectan al estudio detallado de la estacionalidad estocástica de la serie bajo estudio, que reúne dos características - la frecuencia semanal de las observaciones y la existencia de períodos sin exportación- cuya consideración es imprescindible para recoger apropiadamente la conducta de las exportaciones y, concretamente, la naturaleza del comportamiento estacional de las mismas.

PALABRAS CLAVE: exportación de tomates, estacionalidad, raíces unitarias, modelos estructurales, outliers.

CÓDIGOS JEL: Q17

\section{Canary Island tomato exports: a stable seasonal pattern?}

SUMMARY: This paper studies the evolution of the weekly exports of Canary Island tomatoes after the entry of Spain in the European Union. A first analysis of deterministic components reveals their instability, clearly related to changes in the EU commercial regulations of access to European markets, and the presence of many outliers due to different reasons. These factors must be considered in the study of stochastic seasonality of the series. Furthermore, it is necessary to take into account the weekly frequency data and the existence of periods without exports in order to capture its behaviour and, in particular, the nature of its seasonal component.

KEY WORDS: tomato exports, seasonality, unit roots, structural time series models, outliers.

\section{Introducción}

El cultivo del tomate de exportación en las Islas Canarias cuenta ya con una larga tradición. Desde sus inicios a finales del siglo XIX y, sobre todo, en las últimas décadas del siglo XX, los agentes participantes en esta actividad se han caracterizado por un continuado

\footnotetext{
1 José Juan Cáceres Hernández. Facultad de Ciencias Económicas y Empresariales. Campus de Guajara; Camino La Hornera, s/n. 38071 La Laguna. Santa Cruz de Tenerife. Teléfono:(922) 317035. Fax: (922) 317042 e-mail: jcaceres@ull.es
} 
esfuerzo innovador en sus estructuras productivas, así como por la introducción constante de mejoras en el empaquetado y la comercialización. El elemento motriz de este proceso modernizador ha sido la competencia nacional e internacional en los mercados europeos de destino. Sin embargo, además de las transformaciones estructurales, el sostenimiento del producto canario en mercados saturados de ofertas de diferentes orígenes - aunque cada vez más homogéneas en términos de variedades y condiciones de cultivo-, requiere adecuar la oferta a su correspondiente demanda. Dada la elevada incidencia estacional de los volúmenes exportados en la formación de los precios, una distribución adecuada de los envíos a lo largo de la campaña puede evitar bruscas caídas de las cotizaciones y contribuir a maximizar los beneficios de los productores ${ }^{2}$. Desde este punto de vista, el estudio del patrón estacional de la exportación canaria de tomates se torna relevante para la toma de decisiones por parte de los exportadores. En este trabajo se analiza, en concreto, la evolución de las exportaciones semanales de tomate canario desde el ingreso de España en la Unión Europea.

Ahora bien, antes de plantear un modelo estadístico-econométrico que recoja las variaciones experimentadas por esta magnitud, conviene señalar algunas características de la actividad tomatera exportadora del archipiélago. En primer lugar, el patrón estacional de la exportación canaria se ha caracterizado históricamente por la concentración en el invierno y la desaparición en el verano, buscando las épocas de mejores precios. Por otra parte, el desarrollo de los cultivos de invernadero en los países del Norte de Europa, el incremento de la producción peninsular y la aparición de producciones de terceros países que comparten el mismo período exportador que la producción canaria, han determinado un solapamiento creciente de las diferentes ofertas en primavera y otoño. Finalmente, la plena integración española en la PAC, tras un largo período transitorio, ha significado la desaparición de las herramientas con que las producciones europeas se protegían comercialmente frente a la producción canaria ${ }^{3}$, y estos cambios han encontrado una

\footnotetext{
${ }^{2}$ Véase Cáceres (2001).

${ }^{3}$ El sistema de precios de referencia impedía, en la práctica, que la producción canaria accediera a los mercados europeos a partir del mes de abril de cada año. Este sistema fue sustituido por otro más flexible -el sistema de precios de oferta - a partir de julio de 1991. Desde el 1 de enero de 1993 desapareció el sistema de precios de oferta y se liberalizaron las exportaciones canarias a la Comunidad, exceptuando el mantenimiento
} 
respuesta inmediata en los productores de las Islas, a pesar de que los magrebíes han disfrutado también de una reducción notable de las barreras comunitarias ${ }^{4}$.

Si se tienen en cuenta estos aspectos, parece evidente que los volúmenes de tomate canario exportado en las diferentes semanas de la campaña han experimentado variaciones en los últimos años. La cuestión, a efectos de construcción del modelo, estriba en si estas alteraciones ocurren en momentos puntuales que pueden recogerse mediante modificaciones en los componentes determinísticos, o bien, se trata de cambios más continuos y aleatorios que pueden captarse acudiendo a esquemas ARMA con o sin raíces unitarias. Más aún, si se relaja la definición clásica de los tradicionales componentes de una serie temporal, el enfoque de los modelos estructurales se muestra como una herramienta potencialmente apropiada para tratar estas inestabilidades. Por supuesto, este segundo enfoque introduce un nuevo marco conceptual que puede conducir a resultados aparentemente diferentes $y$, sin embargo, equivalentes.

La consideración de estos dos planteamientos metodológicos —que, sin recoger todas las situaciones, amplían el espectro de modelos alternativos y, por tanto, permiten establecer comparaciones-, junto con la presencia de niveles de exportación que se alejan claramente del comportamiento general observado para determinada semana o zafra, las particulares restricciones que aparecen en el tratamiento econométrico de observaciones semanales y la existencia de períodos sin exportación de amplitud variable, son los ingredientes que definen la relevancia analítica de la presente investigación, que se estructura como se indica a continuación. En el epígrafe siguiente se identifican los datos utilizados en el estudio y se comentan algunos aspectos de interés sobre la naturaleza y tratamiento preliminar de éstos. Los resultados del análisis econométrico específico de la evolución de la serie de exportaciones se presentan en los epígrafes tercero y cuarto, en cada uno de los cuales se recurre a una de las dos perspectivas analíticas antes comentadas. El último epígrafe se dedica a exponer las conclusiones del trabajo.

del mecanismo complementario aplicable a los intercambios (MCI), de muy escasa incidencia. La explicación detallada de estos instrumentos de protección puede consultarse en Cáceres (2000: 292-305).

${ }^{4}$ Desde la firma del Protocolo entre Marruecos y la UE en 1988 y hasta la plena integración española en la PAC, la producción magrebí accedía a los mercados europeos en similares condiciones que la producción canaria. Los acuerdos del GATT de 1994 significaban un endurecimiento de las condiciones de acceso de la 


\section{Datos}

En este trabajo se estudia la serie de exportaciones semanales de tomate canario a los mercados europeos, medidas en bultos de $6 \mathrm{~kg}$, en el período posterior al ingreso de España en la Unión Europea, es decir, el comprendido entre las zafras 1986/1987 y 1999/2000 5 . Se considera que cada campaña se inicia en la semana 27 de un año y termina en la semana 26 del año siguiente. Los datos de la serie, en adelante denotada por $\left\{\mathrm{X}_{\mathrm{t}}\right\}_{\mathrm{t}=1, \ldots, 728}$, se representan en la figura $1^{6}$.

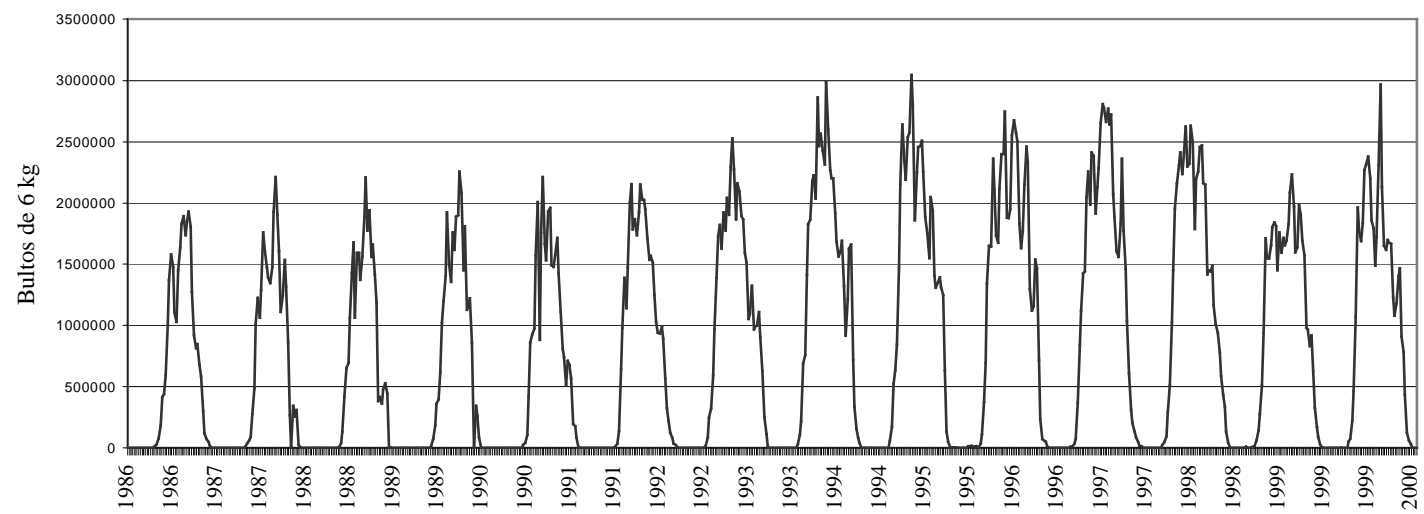

Figura 1. Exportaciones de tomate canario a Europa (1986/1987 a 1999/2000)

El tratamiento de esta serie semanal exige atacar problemas de índole metodológica, ya que a lo largo de un año no existen exactamente 52 semanas, por lo que una semana de un año y la misma semana del año siguiente no recogen exactamente el mismo período del año. Por este motivo, en determinados años pueden incluirse observaciones correspondientes a 53 semanas, lo que introduce un problema de heterogeneidad. Dado que en las series analizadas existía un largo período - el verano- sin observaciones, y

producción marroquí, pero estas condiciones quedaron sin efecto en virtud de los acuerdos comerciales UEMarruecos de 1995 y 1996. Véase Cáceres (2000: 278-281, 308-312).

${ }^{5}$ Los datos se han obtenido de las asociaciones provinciales de cosecheros-exportadores de Santa Cruz de Tenerife (ACETO) y Las Palmas (FEDEX). Las exportaciones a Europa se han calculado como resultado de la suma de las exportaciones al Reino Unido y las dirigidas al resto del Continente. En las semanas en las que estas fuentes no registraban dato alguno, se ha asignado un valor nulo.

${ }^{6} \mathrm{La}$ periodicidad semanal de las observaciones es necesaria para poder descubrir ciertos aspectos del comportamiento estacional de la magnitud económica analizada que podrían quedar ocultos como consecuencia, por ejemplo, de la agregación temporal implícita si se optara por datos mensuales. Por otra parte, las asociaciones de exportadores registran semanalmente sus envíos al exterior, lo que puede 
teniendo en cuenta que en los años con 53 semanas la zafra parecía comenzar una semana más tarde, se ha decidido adelantar una semana el inicio de zafra para estos años.

\section{Estacionalidad determinística y raíces unitarias}

El análisis detallado de la evolución de la serie permite apreciar que en todas las campañas el patrón estacional de exportación se caracteriza por un movimiento ascendente, que se inicia en octubre y que llega hasta enero-febrero, seguido de otro descendente hasta mayo o junio. Y, desde luego, no se observa que los veranos se conviertan en inviernos, como podría ocurrir si el comportamiento estacional estuviese dominado por un componente estocástico no estacionario ${ }^{7}$. Ahora bien, es posible distinguir tres períodos diferenciados por la extensión de la zafra y, sobre todo, por el volumen semanal exportado en determinadas semanas de la campaña. La plena integración en la UE el 1 de enero de 1993, y la consecuente desaparición de los precios de referencia/oferta, se traduce en un notable impulso exportador. El crecimiento general de la exportación en este segundo período se trunca a partir de 1996, coincidiendo con la entrada en vigor del acuerdo comercial entre la UE y Marruecos ${ }^{8}$.

Teniendo en cuenta los cambios observados en el ritmo de crecimiento y en los niveles semanales medios de exportación, así como la aparición en determinadas semanas de valores atípicos, en este apartado se estiman los componentes determinísticos antes de afrontar el análisis del componente estocástico no estacionario de la estacionalidad ${ }^{9}$.

\footnotetext{
interpretarse como indicio de que la semana es un período de tiempo que los agentes económicos consideran adecuado para percibir las reacciones del mercado y poder actuar en consecuencia.

${ }^{7}$ Como indica Hylleberg (1992: 4), la estacionalidad no tiene que ser perfectamente periódica y estable. De hecho, existen tantas vertientes en la naturaleza de la estacionalidad que es difícil aplicar una definición precisa, resultando más factible admitir la posibilidad de recogerla a través de diferentes modelos. Algunos de los trabajos en los que se discuten modelos alternativos del componente estacional son, entre otros, Franses (1994, 1996, 1997), Ghysels (1994), Hylleberg (1986, 1992, 1994) y Miron (1994). Sin embargo, la mayor parte de la literatura econométrica no hace referencia explícita a formulaciones idóneas del componente estacional en el caso de series observadas con frecuencia superior a la mensual. Algunos trabajos en los que se examina el componente estacional en series de alta frecuencia son Koopman (1992) y Harvey, Koopman y Riani (1995).

${ }^{8}$ Un examen de los componentes determinísticos de la serie estudiada en el período comprendido entre las zafras 1986/1987 y 1995/1996 puede consultarse en Cáceres (2001).

${ }^{9}$ Por supuesto, la especificación y estimación previa de los componentes determinísticos puede crear una falsa apariencia de estacionariedad en presencia de raíces unitarias (véase Abeysinghe, 1991, 1994), pero tampoco debe ignorarse que la inconstancia de tales componentes puede motivar la aparición de falsas raíces unitarias (véase Perron y Vogelsang, 1992a, 1992b y Franses y Vogelsang, 1995). Esta circunstancia obligaría a considerar componentes determinísticos inestables en la regresión auxiliar del contraste de integración y, dado que la distribución de los estadísticos de contraste se ve alterada por los componentes determinísticos presentes, sería necesario obtener nuevos valores críticos para la situación considerada.
} 


\subsection{Componentes determinísticos}

El punto de partida para la estimación de los componentes determinísticos radica en la imposición de ciertas hipótesis de cambio estructural. Como se ha comentado, pueden distinguirse tres períodos, caracterizados por distintos niveles medios semanales y ritmos de crecimiento de las exportaciones. Para recoger los diferentes niveles según la semana se han empleado variables cualitativas estacionales, $D_{j}, j=1, \ldots, 52$, que toman el valor 1 si la observación corresponde a la semana j y el valor 0 en otro caso. $\mathrm{Y}$, por otro lado, se ha definido una variable tendencia, T, que va creciendo de forma unitaria desde 1 hasta 728. Se supone, además, que en cada período los ritmos de crecimiento difieren según la semana considerada. De acuerdo con las hipótesis anteriores, los componentes determinísticos finalmente contemplados fueron los siguientes.

Período I: semanas 27/1986-52/1992 (338 observaciones)

En este período sólo existen exportaciones regularmente desde la semana 41 hasta la semana 20. Además, las exportaciones más importantes se concentran entre las semanas 44 y 18 y son, precisamente, las que más crecen. Los niveles y el crecimiento de las exportaciones se recogieron a través de los coeficientes de regresión de las variables cualitativas estacionales $D_{j}, j=41, \ldots, 52,1, \ldots, 20$, y de las variables tendencia T2 у T3, definidas a partir de la variable $\mathrm{T} 1=\mathrm{T}-338^{10}$. Entonces, $\mathrm{T} 2$ y T3 toman el mismo valor que T1 si la observación corresponde a las semanas $44, \ldots, 52,1, \ldots, 18$ y a las semanas $41,42,43$, 19 y 20 del período I, respectivamente, y el valor 0 en cualquier otro caso.

Período II: semanas 1/1993-52/1995 (156 observaciones)

En este período aparecen ya exportaciones regulares desde la semana 40 hasta la 24. Además, se aprecia un crecimiento que es mayor en las semanas con exportaciones más significativas. Para recoger las diferencias en los niveles de exportación antes y después de la plena integración de Canarias en la UE, es decir, entre el período I y el período II, es conveniente definir una variable cualitativa, E193, que toma el valor 1 si la observación pertenece al período II y el valor 0 en otro caso. Con ayuda de esta variable, pueden

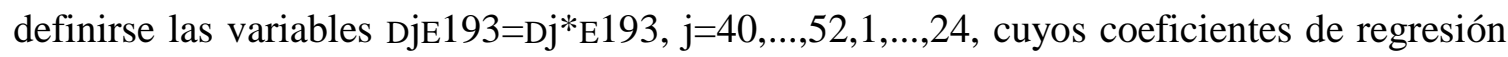
indican el incremento que tendría que experimentar el nivel de la semana $\mathrm{j}$ para que no

\footnotetext{
${ }^{10}$ Este centrado de la tendencia permite evaluar, a través de los coeficientes estimados para las variables cualitativas estacionales, los niveles de exportación estimados en el momento inmediatamente anterior al punto de cambio estructural.
} 
existiese cambio estructural en el nivel de dicha semana en el período II con respecto al período I. Además del salto de nivel, el crecimiento semanal de la exportación en el período II varía según la semana de acuerdo con los coeficientes estimados para las variables tendencia T4 y T5, que toman el mismo valor que T1 si la observación corresponde a las semanas $43, \ldots, 52,1, \ldots, 20$ y $40,41,42,21,22,23$ y 24 del período II, respectivamente, y el valor 0 en cualquier otro caso.

Período III: semanas 1/1996-26/2000 (234 observaciones)

En este período existen exportaciones regulares desde la semana 39 hasta la 24. Sin embargo, se aprecia un cierto decrecimiento que es más intenso en las semanas con exportaciones más significativas. Para recoger las diferencias en los niveles de exportación con respecto al período I, se define la variable E196, que toma el valor 1 si la observación pertenece al período III, y el valor 0 en otro caso, y, a partir de ella, las variables DjE196=Dj*E196, j=39,..,52,1,...24. Además del salto de nivel, el crecimiento semanal de la exportación en el período III viene dado por los coeficientes estimados para las variables T6 y T7, que toman el mismo valor que T1 si la observación corresponde a las semanas $43, \ldots, 52,1, \ldots, 20$ y $39, \ldots, 42,21, \ldots, 24$ del período III, respectivamente, y el valor 0 en cualquier otro caso.

En cada uno de estos tres períodos, las exportaciones observadas en alguna semana no comprendida entre la 41 y la 20 , la 40 y la 24 o la 39 y la 24 , respectivamente, fueron recogidas como observaciones anómalas, así como aquéllas que, perteneciendo a los respectivos intervalos, no registraban exportación alguna. En general, también resultará necesario recurrir al análisis de intervención para la correcta especificación del comportamiento de la serie en observaciones discordantes con el resto. Así, las observaciones anómalas detectadas fueron tratadas mediante la incorporación de variables impulso, $\mathrm{I}_{\mathrm{jk}}$, que toman el valor 1 si la observación corresponde a la semana j del año k y el valor 0 en otro caso. Aunque a priori parece difícil pensar que la producción de tomate en Canarias pueda cambiar de una semana a otra, sí existen factores que pueden producir estos cambios $^{11}$ y que explican la aparición de observaciones anómalas con cierta frecuencia ${ }^{12}$.

\footnotetext{
${ }^{11}$ Así, la bondad de las temperaturas en el otoño es el principal responsable del incremento exportador en el principio de zafra de las campañas 93/94, 94/95 y 95/96. No es extraño tampoco que los agricultores aceleren la recogida del fruto en épocas de altos precios, o simplemente, que se descuide algo la selección y se exporten más partidas de las que se hubieran enviado en otras circunstancias. O bien, al contrario, suele ocurrir que la exportación se frena y se selecciona con más rigor en las semanas de cotizaciones bajas.
} 
Para la detección de estas observaciones se han empleado distintos procedimientos estadísticos, sin descuidar la observación directa de la matriz de datos y las representaciones gráficas. De todas formas, es preciso aceptar que la selección de éstas, y no otras observaciones, como anómalas es, al menos hasta cierto punto, subjetiva, ya que depende de la veracidad de las hipótesis impuestas sobre los cambios en los componentes determinísticos ${ }^{13}$.

Todos estos componentes determinísticos han sido estimados por diferentes métodos, bajo el supuesto de estacionariedad. En primer lugar, se realizó la estimación por mínimos cuadrados ordinarios del siguiente modelo de regresión lineal (método 1):

$$
\begin{aligned}
& X_{t}=\sum_{j=41}^{52} \alpha_{j} D j+\sum_{j=1}^{20} \alpha_{j} D j+\sum_{j=40}^{52} \beta_{j} D j E 193+\sum_{j=1}^{24} \beta_{j} D_{j} E 193+\sum_{j=39}^{52} \gamma_{j} D j E 196+ \\
& +\sum_{j=1}^{24} \gamma_{j} D_{j} E 196+\delta_{2} T 2+\delta_{3} T 3+\delta_{4} \mathrm{~T} 4+\delta_{5} \mathrm{~T} 5+\delta_{6} \mathrm{~T} 6+\delta_{7} \mathrm{~T} 7+\sum_{j, k} \lambda_{j k} I_{j k}+\varepsilon_{t}
\end{aligned}
$$

siendo $\varepsilon_{\mathrm{t}}$ un término de perturbación aleatoria que se supone, a priori, ruido blanco.

Sin embargo, el análisis univariante de la serie de residuos de este modelo muestra que las perturbaciones no son esféricas. Por un lado, la matriz de varianzas-covarianzas de los elementos del vector de perturbaciones toma valores distintos de cero fuera de la diagonal principal, es decir, existen problemas de autocorrelación. Pero, además, los grupos de observaciones nulas entre las campañas pueden provocar heteroscedasticidad ${ }^{14}$.

Se ha intentado solucionar el problema de autocorrelación residual acudiendo a un método de estimación en dos etapas (método 2). La primera consiste en obtener la serie de residuos de la estimación de los componentes determinísticos por el método 1 y estimar para dichos residuos un esquema autorregresivo. En la segunda etapa, los retardos de los

\footnotetext{
12 Un 10\%, aproximadamente, de las observaciones se detectaron como anómalas. Algunas de ellas correspondían a semanas en las que normalmente no había exportación o, por el contrario, ésta no existía en semanas habituales de zafra.

${ }^{13}$ Además, suele ser difícil distinguir si una vez observado un valor extremo, éste es una observación anómala o una observación regular asociada con la naturaleza inherente a la propia serie, cuyo comportamiento puede responder a un modelo más o menos complejo.

${ }^{14}$ La correlación serial se detectó por medio de los estadísticos de multiplicadores de Lagrange de Breusch y Godfrey y los estadísticos Q de Ljung y Box. La heteroscedasticidad se estudió mediante el test de Breusch y Pagan, suponiendo que la variable J (semana) era la relacionada con la heteroscedasticidad.
} 
residuos de la primera que aparecían como significativos se introdujeron como variables independientes en la regresión de estimación de los componentes determinísticos ${ }^{15}$.

\section{CUADRO 1}

Estimaciones de los ritmos de crecimiento semanal (períodos I, II y III)

\begin{tabular}{|l|c|c|c|c|c|}
\hline T2: 1566.418 & T3: 475.876 & T4: 1993.682 & T5: 102.311 & T6: -1637.08 & T7: -476.021 \\
\hline
\end{tabular}

\section{CUADRO 2}

Estimaciones de los niveles semanales (períodos I, II y III)

\begin{tabular}{|c|c|c|c|c|c|c|c|}
\hline D41 & 42491.263 & D49 & 1675035 & D5 & 2212756.6 & D13 & 998932.92 \\
\hline D42 & 106125.33 & D50 & 1899872.3 & D6 & 2312184.4 & D14 & 774229.93 \\
\hline $\mathrm{D} 43$ & 143144.58 & D51 & 2171171.3 & D7 & 2231489.2 & D15 & 716539.05 \\
\hline D44 & 340459.9 & D52 & 1842622.5 & D8 & 1797563.4 & D16 & 791940.57 \\
\hline $\mathrm{D} 45$ & 656054.41 & D1 & 1837211.3 & D9 & 1836704.9 & D17 & 709544.61 \\
\hline D46 & 936811.72 & D2 & 1829193.7 & D10 & 1585771.7 & D18 & 419197.23 \\
\hline D47 & 1162861.6 & D3 & 1801823.1 & D11 & 1535326.1 & D19 & 206938.57 \\
\hline $\mathrm{D} 48$ & 1331696.6 & D4 & 2110785.8 & D12 & 1381536 & D20 & 111027.69 \\
\hline D40E193 & 16034.913 & $\overline{\mathrm{D} 50 \mathrm{E} 193}$ & 129641.48 & D7E193 & -58937.25 & D16E193 & 152664.32 \\
\hline D41E193 & 32335.365 & D51E193 & -154424.4 & D8E193 & 269440.53 & D17E193 & 364830.43 \\
\hline D42E193 & 98981.72 & D52E193 & 589047.23 & D9E193 & 37928.802 & $\mathrm{D} 18 \mathrm{E}$ & 795657.3 \\
\hline D43E193 & 126981 & D1E193 & 278819.39 & D10E193 & 147156.3 & D19E & 979812 \\
\hline D44E193 & 356883.17 & D2E193 & 7133 & D11E1 & -87078.11 & D20E1 & 508332.68 \\
\hline D45E193 & 73634.942 & D3E193 & 497151.35 & D12E193 & 190249.57 & D21E193 & 377486.12 \\
\hline D46E193 & 351122.85 & D4E193 & 161026.57 & D13E193 & 420114.7 & D22E193 & 148415.17 \\
\hline D47E193 & 744493.04 & D5E193 & 70215.243 & D14E193 & 491789.36 & D23E193 & 67412.441 \\
\hline D48E193 & 483059.4 & D6E193 & -168448.1 & D15E193 & 479209.84 & D24E193 & 17249.229 \\
\hline D49E193 & 465854.7 & & & & & & \\
\hline D39E196 & 117724.51 & D49E196 & 96316 & & 9.32 & D16E196 & 907158.78 \\
\hline D40E196 & 159108.31 & D50E196 & 632514.36 & D8E196 & 1102517.2 & D17E196 & 778447.63 \\
\hline D41E196 & 212960.93 & D51E196 & 651613.52 & D9E196 & 939817.56 & D18E196 & 1084324 \\
\hline D42E196 & 255636.65 & D52E196 & 979295.78 & D10E196 & 719051.6 & D19E196 & 1270064.3 \\
\hline D43E196 & 775532.58 & D1E196 & 635406.41 & D11E196 & 741885.53 & D20E196 & 998455.34 \\
\hline D44E196 & 1079431.2 & D2E196 & 677957.09 & D12E196 & 811310.82 & D21E196 & 523395.16 \\
\hline D45E196 & 1346282.3 & D3E196 & 495931.86 & D13E196 & 1073438.9 & D22E196 & 336592.77 \\
\hline D46E196 & 1197309.4 & D4E196 & 126910.99 & D14E196 & 1215347.1 & D23E196 & 234843.7 \\
\hline D47E196 & 1097225.5 & D5E196 & 33454.67 & D15E196 & 1280339.9 & D24E196 & 192868.53 \\
\hline D48E196 & 1118107.6 & D6E196 & 558120.51 & & & & \\
\hline
\end{tabular}

Para paliar el problema de heteroscedasticidad generado por las observaciones correspondientes a semanas con exportaciones nulas durante el verano, se optó por eliminar

\footnotetext{
${ }^{15}$ En este método, la serie de exportaciones utilizada para el posterior estudio de raíces unitarias ha sido filtrada por las intervenciones estimadas en la etapa 1 para la campaña 86/87, ya que estas intervenciones no podían estimarse en la etapa 2 debido a la incorporación de retardos. Un procedimiento teóricamente más adecuado para la estimación de los componentes determinísticos cuando el término de perturbación presenta autocorrelación consiste en la estimación conjunta, por máxima verosimilitud, de dichos componentes y del modelo ARMA apropiado para el componente residual. Sin embargo, las limitaciones de capacidad de los programas informáticos disponibles (se intentó la estimación con el SCA) impedían su aplicación.
} 
dichas observaciones ${ }^{16}$ y efectuar dos tipos de estimaciones: sin considerar la estructura de correlación del término de perturbación (método 3) y considerando como regresores los retardos de los residuos estimados en el método anterior (método 4). Los tests de autocorrelación y heteroscedasticidad mostraban la ausencia de problemas en este último método, cuyas estimaciones se muestran en los cuadros 1 y $2^{17}$.

\subsection{Análisis de integración}

Los tests de raíz unitaria se verán sensiblemente afectados por el modelo planteado para los componentes determinísticos. Por esta razón y aunque, como se ha señalado, algunos de los métodos de estimación empleados son preferibles a otros, se ha optado por eliminar los componentes determinísticos estimados por todos los métodos anteriores y obtener así series sin dichos componentes, $\left\{\mathrm{Fx} 1_{\mathrm{t}}\right\},\left\{\mathrm{Fx} 2_{\mathrm{t}}\right\}$ y $\left\{\mathrm{Fx} 4_{\mathrm{t}}\right\}$, sobre las que se efectuó el análisis de integración ${ }^{18}$. En caso de conflicto entre los resultados para unas y otras, la balanza se inclinará hacia los obtenidos a partir de las estimaciones del método 4.

Se han efectuado los contrastes de integración estacional desarrollados para el caso semanal en la línea de Hylleberg y otros $(1990)^{19}$. Para resolver el problema de correlación en la regresión auxiliar de contraste, se ha utilizado también la estrategia propuesta por Hylleberg y otros (1990), que consiste en añadir como regresores los retardos necesarios de la variable dependiente hasta blanquear los residuos ${ }^{20}$. Es decir, se estima la regresión

$$
\mathrm{Y}_{28, \mathrm{t}}=\mu_{\mathrm{t}}+\pi_{1} \mathrm{Y}_{1, \mathrm{t}-1}+\pi_{2} \mathrm{Y}_{2, \mathrm{t}-1}+\sum_{\mathrm{k}=3}^{27}\left[\pi_{\mathrm{k}, 1} \mathrm{Y}_{\mathrm{k}, \mathrm{t}-1}+\pi_{\mathrm{k}, 2} \mathrm{Y}_{\mathrm{k}, \mathrm{t}-2}\right]+\sum_{\mathrm{m}} \phi_{\mathrm{m}} \mathrm{Y}_{28, \mathrm{t}-\mathrm{m}}+\varepsilon_{\mathrm{t}}
$$

donde: $Y_{1, t}=\frac{\left(1-B^{52}\right)}{1-B} X_{t} ; Y_{2, t}=-\frac{\left(1-B^{52}\right)}{1+B} X_{t}$

$$
\mathrm{Y}_{\mathrm{k}, \mathrm{t}}=-\frac{1-\mathrm{B}^{52}}{\left[1-2 \cos \left(\frac{2(\mathrm{k}-2) \pi}{52}\right) \mathrm{B}+\mathrm{B}^{2}\right]} \mathrm{X}_{\mathrm{t}}, \mathrm{k}=3,4, \ldots, 27 ; \mathrm{Y}_{28, \mathrm{t}}=\left(1-\mathrm{B}^{52}\right) \mathrm{X}_{\mathrm{t}}
$$

\footnotetext{
${ }^{16}$ En la semana 14 de 1990 la exportación es nula, pero se trata claramente de una observación anómala ya que la zafra no había terminado aún y, por lo tanto, no fue eliminada.

${ }^{17}$ Las estimaciones obtenidas por los diferentes métodos son similares entre sí (las del método 3 coinciden con las del método 1) y, por tanto, se ha decidido mostrar sólo los resultados del método 4.

${ }^{18}$ Las series filtradas de los componentes determinísticos estimados por el método 3 no se han considerado ya que coinciden con las estimaciones del método 1.

${ }^{19}$ Véase Cáceres (1996).

${ }^{20}$ Antes de realizar los contrastes de integración propiamente dichos, se ha aplicado la metodología ARIMA tradicional, que revela que es posible que las series, sin diferencias regular ni estacional, sigan procesos que pueden formularse como autorregresivos sin excesivas complicaciones.
} 
Nótese que en la regresión de contraste aparece el término $\mu_{\mathrm{t}}$, que recoge los componentes determinísticos. Dado que las series $\left\{x_{t}\right\}$ sobre las que se aplicarán los contrastes son $\left\{\mathrm{Fx} 1_{\mathrm{t}}\right\},\left\{\mathrm{Fx} 2_{\mathrm{t}}\right\}$ y $\left\{\mathrm{Fx} 4_{\mathrm{t}}\right\}$, se espera que en esta regresión estos términos no sean significativos. Aún así, puede que estos componentes no hayan sido correctamente eliminados en todos los métodos, por lo que podrían obtenerse conclusiones erróneas. Por ello, se ha estimado la regresión considerando diferentes hipótesis sobre el término $\mu_{\mathrm{t}}$ : ausencia de componentes determinísticos, constante, constante y tendencia, constante y variables cualitativas estacionales, y constante, tendencia y variables cualitativas estacionales. Para cualquiera de las tres series analizadas e independientemente de los componentes determinísticos considerados en la regresión auxiliar de contraste, los resultados de este procedimiento (cuadro 3 ) $^{21}$ revelan la ausencia de integración tanto en las frecuencias estacionales como en la frecuencia cero ${ }^{22}$.

El resultado obtenido con respecto a las frecuencias estacionales concuerda con la observación de los gráficos por campaña ${ }^{23}$, en los que se aprecia un cambio en los niveles de exportación como consecuencia de la nueva normativa comercial que regula las exportaciones de tomate desde Canarias a la UE; pero, además de ese cambio, el patrón estacional es más o menos regular, con máximos y mínimos localizados casi siempre en torno a las mismas semanas de la zafra, sin que se detecten cambios aleatorios en cualquier dirección. En otras palabras, parece que el comportamiento estacional de las exportaciones canarias a Europa está dominado por los componentes determinísticos. Ahora bien, esta conclusión debe ser tomada con la necesaria cautela, al menos por dos razones. Por un lado, porque el hecho de que el valor de la serie en una determinada estación no experimente grandes cambios aleatorios no es evidencia suficiente para rechazar la existencia de raíces

\footnotetext{
${ }^{21}$ Sólo se muestran los valores de los estadísticos de contraste para la serie $\{\mathrm{Fx} 4\}$ sin incluir en la regresión auxiliar componentes determinísticos, que, como cabe esperar, no resultan significativos. En el caso particular de las frecuencias asociadas a pares de raíces unitarias complejas conjugadas, se ha preferido adoptar la decisión sobre la existencia o no de raíz unitaria a partir de los valores del estadístico $\mathrm{F}$ de significación del par de raíces unitarias. Nótese que no es posible concluir que existe sólo una de las dos raíces unitarias complejas conjugadas, ya que la serie objeto de estudio es una serie de observaciones reales. Por otro lado, los estudios de Ghysels y otros (1994) demuestran, para el caso trimestral, que el test conjunto es preferible al contraste secuencial basado en los estadísticos t de significación individual.

${ }^{22}$ Dados los resultados obtenidos y el propio comportamiento observado para la serie, se descartó la posibilidad de múltiples raíces unitarias en alguna de las frecuencias estacionales, de modo que no se consideró necesario recurrir a la estrategia de contraste propuesta por Franses y Taylor (2000).

${ }^{23}$ Estos gráficos no se muestran aquí por restricciones de espacio.
} 
unitarias estacionales ${ }^{24}$. Y, por otro, porque los resultados obtenidos dependen de las hipótesis establecidas sobre los cambios en los componentes determinísticos ${ }^{25}$.

A pesar de la claridad de los resultados de la estrategia de contraste antes comentada con respecto a la frecuencia cero, los tests de Dickey-Fuller $(\mathrm{DF})^{26}$ pueden ayudar a confirmar la conclusión sobre la presencia de esta raíz unitaria. La existencia de autocorrelación en los residuos de la regresión auxiliar del test DF obliga a utilizar el test de Dickey-Fuller Aumentado $(\mathrm{ADF})^{27}$. Además, con respecto a los componentes determinísticos, se ha desarrollado la estrategia de contraste como si éstos estuvieran presentes.

\section{CUADRO 3}

Estadísticos para el contraste de integración estacional (serie $\{\mathbf{F x} 4\}$ )

\begin{tabular}{|c|c|c|c|c|c|c|c|}
\hline $\mathrm{LBQ}(1)=0.26$ & $\mathrm{LBQ}(52)=12.85$ & $\mathrm{LBQ}(104)=43.72$ & \multicolumn{3}{|c|}{$\mathrm{B}-\mathrm{G}(1)=2.14$} & \multicolumn{3}{c|}{$\mathrm{B}-\mathrm{G}(52)=33.83$} & $\mathrm{~B}-\mathrm{G}(104)=77.63$ \\
\hline $\mathrm{t} 1$ & -6.455716 & F6 & 48.75489 & F13 & 36.74556 & F20 & 23.24502 \\
\hline $\mathrm{t} 2$ & -4.465283 & F7 & 31.90947 & F14 & 21.20464 & F21 & 24.93109 \\
\hline F1 & 41.39923 & F8 & 13.79335 & F15 & 23.12235 & F22 & 30.53466 \\
\hline F2 & 23.01672 & F9 & 22.07308 & F16 & 25.02561 & F23 & 30.62357 \\
\hline F3 & 19.66220 & F10 & 35.30517 & F17 & 24.60277 & F24 & 25.99071 \\
\hline F4 & 52.48639 & F11 & 49.97052 & F18 & 23.06620 & F25 & 24.76643 \\
\hline F5 & 45.69138 & F12 & 59.67356 & F19 & 34.99821 & F26 & 12.53251 \\
\hline
\end{tabular}

Nota: Los retardos incorporados en la regresión auxiliar son: 1, 6, 8, 9, 51, 52, 53, 58, 61, 104.

En realidad, el filtrado previo de los componentes determinísticos se manifestó en la no significación de estos componentes. En todos los casos, el decrecimiento lento de los coeficientes estimados para la formulación autorregresiva mostraba con claridad que la serie doblemente diferenciada estaba sobrediferenciada, resultado que confirmaba el test $\mathrm{ADF}$, que conducía al rechazo de la hipótesis de raíz unitaria frente a la alternativa

\footnotetext{
${ }^{24}$ Téngase en cuenta que si las varianzas del proceso con raíz unitaria son poco importantes en relación con el nivel de la serie, se producirán cambios lentos pero permanentes.

${ }^{25}$ A pesar de que los contrastes anteriores llevan al rechazo de la hipótesis de integración en las frecuencias estacionales, existe la posibilidad de que el filtrado previo de los componentes determinísticos - y, en particular, de las variables cualitativas estacionales-, esté incidiendo en la obtención de resultados a favor de la hipótesis alternativa de estacionariedad. Para tratar de responder el interrogante generado, podría obtenerse la serie filtrada de todos los componentes determinísticos excepto los niveles estimados a finales del período I. Sin embargo, la rotundidad de los resultados obtenidos hace difícil pensar que la conclusión pueda cambiar mediante este procedimiento.

${ }^{26}$ Véase Fuller (1976) y Dickey y Fuller (1981).

${ }^{27}$ Por otra parte, y atendiendo a las recomendaciones de Dickey y Pantula (1987), se ha aplicado un contraste en tres etapas. Primero se contrasta si la serie doblemente diferenciada posee aún una raíz unitaria en la frecuencia cero. Si se rechaza esta hipótesis, se pasa a contrastar si la serie diferenciada posee raíz unitaria en dicha frecuencia. Si también se rechaza la hipótesis contrastada, se pasa finalmente a contrastar si la serie sin diferenciar posee o no raíz unitaria.
} 
estacionaria fueran cuáles fuesen los componentes determinísticos incluidos en la regresión auxiliar de contraste. En el cuadro 4 sólo se indican los valores del estadístico ADF para la serie $\{\mathrm{Fx} 4\}$ sin incluir componentes determinísticos.

Cabe pensar que unos resultados tan claros sobre la ausencia de raíz unitaria en la frecuencia cero, pueden ser explicados por la existencia de grupos de observaciones nulas entre campañas. Con el propósito de contrastar si en el resto de la serie el comportamiento presentaba el mismo carácter estacionario, se decidió aplicar los contrastes ADF sobre la serie $\left\{\mathrm{Fx} 4^{*}\right\}$, construida eliminando las observaciones nulas del verano de la serie $\{\mathrm{Fx} 4\}^{28}$. Los resultados obtenidos para esta serie, que también aparecen en el cuadro 4, siguen mostrando con claridad el rechazo de la hipótesis de raíz unitaria en la frecuencia cero.

\section{CUADRO 4}

\section{Contraste ADF de raíz unitaria en la frecuencia cero}

\begin{tabular}{|c|c|c|c|c|c|c|c|c|}
\hline Series & LBQ(1) & LBQ(52) & LBQ(104) & B-G(1) & B-G(52) & B-G(104) & t & V. crítico \\
\hline FX4 & 0.042889 & 8.46190 & 45.2464 & 0.358994 & 40.9436 & 82.2887 & -5.23501 & -1.9400 \\
\hline FX4* & 0.023894 & 46.6052 & 109.917 & 0.261194 & 58.7331 & 107.957 & -13.9586 & -1.9401 \\
\hline
\end{tabular}

Nota: Los retardos incorporados en la regresión auxiliar son 1 a 51,53 a 60, 104 y 156, para la serie $\{\mathrm{Fx} 4\}$, y $1,6,7,8,26,36$ y 42 , para la serie $\{\mathrm{Fx} 4 *\}$.

Nota: El estadístico t representa el estadístico de Dickey-Fuller para el contraste de la hipótesis de raíz unitaria en el modelo sin componentes determinísticos. Los valores críticos que se señalan han sido obtenidos a partir de la superficie de respuesta calculada por Mackinnon (1991) para un nivel de confianza del $95 \%$.

\section{Modelos estruCturales}

En el epígrafe anterior, la conclusión sobre la estabilidad del patrón estacional de exportación se asienta sobre ciertas hipótesis que asocian los cambios estructurales observados con modificaciones puntuales en la normativa comercial y que, a partir del conocimiento cualitativo del mercado y de la observación de los datos, establecen diferentes ritmos de crecimiento en los envíos según las semanas. En este apartado, se recurre a los modelos estructurales como una metodología capaz de recoger las inestabilidades de los diferentes componentes de la serie mediante criterios estadísticos y sin que el investigador establezca, al menos a priori, patrones de cambio tan definidos como los del apartado anterior.

La mayor disponibilidad de datos en los últimos años y, sobre todo, la posibilidad de construir series más largas y con mayor frecuencia de observación, han estimulado el uso, e incluso el desarrollo, de técnicas estadístico-econométricas. En concreto, para las 
nuevas series resulta cada vez más difícil mantener el supuesto de un patrón de comportamiento fijo a lo largo del tiempo y, en este sentido, los modelos estructurales de series temporales son una técnica apropiada, puesto que admiten la posibilidad de que cada uno de los componentes inobservables típicos de una serie temporal posea una naturaleza estocástica $^{29}$. Así, el modelo estructural básico (BSM) incluye tendencia, con nivel y pendiente estocásticas, estacionalidad trigonométrica, también estocástica, y un componente irregular que recoge movimientos no sistemáticos. Su formulación estadística es $Y_{t}=\mu_{t}+\gamma_{t}+\varepsilon_{t}, \quad$ con $\quad \mu_{t}=\mu_{t-1}+\beta_{t-1}+\eta_{t}, \quad \beta_{\mathrm{t}}=\beta_{\mathrm{t}-1}+\varsigma_{\mathrm{t}} \quad \mathrm{y} \quad \gamma_{t}=\sum_{j=1}^{[s / 2]} \gamma_{j, t}, \quad$ donde $\varepsilon_{t} \sim \mathrm{NID}\left(0, \sigma_{\varepsilon}^{2}\right), \eta_{t} \sim \mathrm{NID}\left(0, \sigma_{\eta}^{2}\right)$ y $\varsigma_{t} \sim \mathrm{NID}\left(0, \sigma_{\varsigma}^{2}\right) . \beta_{t}$ representa la pendiente de la tendencia $\mu_{t}, \gamma_{t}$ recoge el componente estacional y el componente irregular se denota por $\varepsilon_{t}$. Respecto al componente estacional, cada término $\gamma_{j, t}$ es generado por $\left[\begin{array}{c}\gamma_{j, t} \\ \gamma_{j, t}^{*}\end{array}\right]=\left[\begin{array}{cc}\cos \lambda_{j} & \sin \lambda_{j} \\ -\sin \lambda_{j} & \cos \lambda_{j}\end{array}\right]\left[\begin{array}{c}\gamma_{j, t-1} \\ \gamma_{j, t-1}^{*}\end{array}\right]+\left[\begin{array}{c}\omega_{j, t} \\ \omega_{j, t}^{*}\end{array}\right], \quad j=1, \ldots,[s / 2], \quad t=1, \ldots, T$, donde $\gamma_{j}^{*}$ posee un valor instrumental que determina el punto de inicio del movimiento cíclico asociado a la frecuencia estacional $\lambda_{j}=2 \pi j / s$, en radianes, y las perturbaciones $\omega_{\mathrm{j}, \mathrm{t}} \mathrm{y} \omega_{\mathrm{j}, \mathrm{t}}^{*}$ están mutua y serialmente incorreladas y distribuidas NID con media cero y varianza común $\sigma_{\omega}^{2}$. Se supone, además, que el componente irregular, $\varepsilon_{t}$, el término de perturbación del nivel, $\eta_{t}$, la perturbación de la pendiente, $\varsigma_{t}$, y los términos de perturbación de los ciclos estacionales, $\omega_{\mathrm{j}, \mathrm{t}}$ y $\omega_{\mathrm{j}, \mathrm{t}}^{*}$, están mutua y serialmente incorrelados.

La estimación del modelo anterior exige recurrir al denominado filtro de $\operatorname{Kalman}^{30}$, que permite evaluar los diferentes componentes de la serie (variables de estado) en cada uno de los momentos del tiempo. Dadas las dimensiones del espacio de variables de estado, esta información suele recogerse a través de las representaciones gráficas de los componentes en los diferentes instantes del tiempo y, de forma más escueta, en el vector de estado final, que recoge la estimación de cada uno de ellos en el instante de tiempo T.

\footnotetext{
${ }^{28}$ Se han eliminado las mismas observaciones que fueron suprimidas para la estimación de los componentes determinísticos por el método 4.

${ }^{29}$ Una exposición detallada de estos modelos puede encontrarse en Harvey (1989), Harvey (1993) y Durbin y Koopman (2001).

${ }^{30}$ Véase Kalman (1960) y Kalman y Bucy (1961).
} 
Como puede deducirse, todos los componentes del modelo son dirigidos por sus respectivos términos de perturbación. De ahí que el centro de atención en la primera fase de análisis recaiga sobre la estimación de sus varianzas. En términos relativos, la información contenida en las varianzas puede recogerse a través de los ratios señal-ruido (q), definidos como el cociente entre las varianzas de cada uno de los componentes y la del componente de mayor varianza. El modelo debe reespecificarse si, a partir de estas estimaciones, se concluye que alguna de las varianzas de los componentes es nula, es decir, que dicho componente es determinístico o fijo. Una vez aceptada la naturaleza no estocástica de un componente determinado, puede decidirse la conveniencia o no de eliminarlo a partir de contrastes de significación de su valor en el vector de estado final ${ }^{31}$.

\subsection{Examen de la serie de exportaciones}

A pesar de que la representación gráfica de la serie de exportaciones no permite concluir que el patrón estacional sea estocástico, se optó por iniciar la estrategia de identificación de la naturaleza de los componentes mediante la estimación del modelo estructural básico (modelo 1). La estimación máximo verosímil de este modelo ${ }^{32}$ revela que la varianza del componente estacional y de la pendiente son nulas, lo que manifiesta el carácter no estocástico de tales componentes. Este resultado permite evaluar la presencia de dichos componentes en el modelo a partir de los contrastes habituales de significación de su valor correspondiente en el vector de estado final. El estadístico de significación individual de la pendiente permite concluir que el modelo debe simplificarse eliminando esté término en la tendencia. Respecto al componente estacional, a pesar de su comportamiento no estocástico y de la no significación estadística individual del parámetro correspondiente a algunas estaciones, el estadístico de significación conjunta del efecto estacional ${ }^{33}$ parece confirmar la presencia de este componente en el comportamiento de la serie (cuadro 5).

La representación gráfica de los componentes estimados para el modelo anterior resulta sumamente reveladora, dado que refleja el carácter fijo del patrón estacional a lo largo de la muestra, así como la estabilidad de la pendiente en torno a un valor realmente pequeño en relación con la magnitud de las observaciones. Teniendo en cuenta estos

\footnotetext{
${ }^{31} \mathrm{Si}$ se trata de un componente determinístico, la información del estado se reduce a la que proporciona el vector de estado final.

${ }^{32}$ Se ha utilizado la opción de alisado (smoothed) del programa STAMP 6.0 (véase Koopman y otros, 2000).
} 
resultados, se plantea un modelo en el que el patrón estacional es fijo, pero, con objeto de examinar las implicaciones de diferentes modelos para la tendencia, se permite que cada uno de sus elementos (nivel y pendiente) mantenga su formulación estocástica (modelo 2): $Y_{t}=\mu_{t}+\gamma_{t}+\varepsilon_{t}$, con $\mu_{t}=\mu_{t-1}+\beta_{t-1}+\eta_{t}, \beta_{\mathrm{t}}=\beta_{\mathrm{t}-1}+\varsigma_{\mathrm{t}}$ y $\gamma_{\mathrm{t}}=\sum_{\mathrm{j}=1}^{51} \mathrm{z}_{\mathrm{j}, \mathrm{t}} \gamma_{\mathrm{j}}$, siendo $z_{j, t}, \mathrm{j}=1, \ldots$, 51, igual a 1 si la observación pertenece a la estación j, -1 si la observación pertenece a la estación 52 y 0 en otro caso. De nuevo, el proceso de estimación conduce a la conclusión de que la varianza del término de perturbación que dirige el comportamiento de la pendiente es nula y el estadístico de significación individual en el vector de estado final manifiesta su no significación estadística (cuadro 6). La representación gráfica de los componentes del modelo estimado es, como cabe esperar, muy similar a la del modelo inicial.

\section{CUADRO 5}

Estimación de los parámetros del modelo 1

\begin{tabular}{|l|c|c|c|c|}
\hline \multicolumn{5}{|c|}{ Estimación de las varianzas de los componentes } \\
\hline Componentes & Irregular & Nivel & Pendiente & Estacionalidad \\
\hline Varianza & $2.6302 \mathrm{e}+010$ & $4.9262 \mathrm{e}+009$ & 0.00000 & 0.00000 \\
\hline Ratio Señal-Ruido & $(1.0000)$ & $(0.1873)$ & $(0.0000)$ & $(0.0000)$ \\
\hline \multicolumn{4}{|c|}{ Significación de los componentes en el vector de estado final } \\
\hline Test t de significación individual & \multicolumn{2}{c|}{ Nivel: 71.598} & Pendiente: -0.052363 \\
\hline Test de significación conjunta del efecto estacional & \multicolumn{2}{c|}{501.519} \\
\hline
\end{tabular}

\section{CUADRO 6}

Estimación de los parámetros del modelo 2

\begin{tabular}{|l|c|c|c|c|}
\hline \multicolumn{5}{|c|}{ Estimación de las varianzas de los componentes } \\
\hline Componentes & Irregular & Nivel & Pendiente & Estacionalidad \\
\hline Varianza & $5.8460 \mathrm{e}+009$ & $3.9135 \mathrm{e}+010$ & 0.00000 & - \\
\hline Ratio Señal-Ruido & $(0.1494)$ & $(1.0000)$ & $(0.0000)$ & - \\
\hline \multicolumn{4}{|c|}{ Test t de significación individual de nivel y pendiente en el vector de estado final } \\
\hline \multicolumn{4}{|c|}{ Nivel: 64.113 } & \multicolumn{3}{|c|}{ Pendiente: -0.00045166 } \\
\hline
\end{tabular}

Nota: No se incluyen los test de significación individual para el componente estacional porque los resultados son similares a los del modelo finalmente estimado.

Aceptando que el patrón estacional permanece inalterado con el transcurso del tiempo y que la pendiente es nula, se especificó el modelo 3: $Y_{t}=\mu_{t}+\gamma_{t}+\varepsilon_{t}$, con

\footnotetext{
${ }^{33}$ Este estadístico, que evalúa el efecto conjunto del componente estacional, sigue una distribución $\chi^{2}$ con (s-1) grados de libertad, 51 en nuestro caso al tratarse de las exportaciones semanales.
} 
$\mu_{t}=\mu_{t-1}+\eta_{t} \quad$ y $\quad \gamma_{\mathrm{t}}=\sum_{\mathrm{j}=1}^{51} \mathrm{z}_{\mathrm{j}, \mathrm{t}} \gamma_{\mathrm{j}}$. Los resultados obtenidos para este modelo ratifican el carácter estocástico del componente de nivel (cuadro 7). A partir de estas estimaciones se procedió a obtener la serie ajustada estacionalmente con objeto de facilitar la observación del comportamiento tendencial y de posibles observaciones anómalas ${ }^{34}$. El gráfico de la serie ajustada de estacionalidad, si se compara con el del componente tendencial del modelo 3, revela que el nivel estocástico recoge bien el comportamiento de la serie en cuestión y, por tanto, nos lleva de nuevo a concluir que el modelo estructural que parece adecuado debe incluir un componente de nivel estocástico, estacionalidad fija y un componente irregular.

\section{CUADRO 7}

Estimación de los parámetros del modelo 3

\begin{tabular}{|l|c|c|c|c|}
\hline \multicolumn{5}{|c|}{ Estimación de las varianzas de los componentes } \\
\hline Componentes & Irregular & Nivel & Pendiente & Estacionalidad \\
\hline Varianza & $6.0772 \mathrm{e}+009$ & $3.8655 \mathrm{e}+010$ & - & - \\
\hline Ratio Señal-Ruido & $(0.1572)$ & $(1.0000)$ & - & - \\
\hline \multicolumn{7}{|c|}{ Test de significación } \\
\hline
\end{tabular}
Test de significación individual del componente de nivel en el vector de estado final: 64.064 Nota: No se incluyen los test de significación individual para el componente estacional porque los resultados son similares a los del modelo finalmente estimado.

Pero antes de adoptar conclusiones definitivas sobre la bondad del modelo, deben examinarse el componente estacional, las innovaciones o errores de predicción y los residuos de los dos componentes estocásticos. Respecto al componente estacional, los estadísticos de significación individual en el vector de estado final revelan el carácter significativamente distinto de cero para la mayoría de las semanas ${ }^{35}$. Por otra parte, puede asumirse que los errores estandarizados de predicción se comportan adecuadamente como $\mathrm{NID}(0,1)$. Ahora bien, el examen de los residuos en cada momento del tiempo y, especialmente, su representación gráfica muestran, como de hecho podía haberse observado ya, la presencia de observaciones anómalas tales como la detectada en la semana 51 de 1990. La presencia de outliers exige reespecificar el modelo en los términos siguientes:

\footnotetext{
${ }^{34}$ La serie ajustada de estacionalidad se obtiene como $\mathrm{Y}_{\mathrm{t}}-\widetilde{\gamma}_{\mathrm{t}}$, donde $\widetilde{\gamma}_{\mathrm{t}}$ representa la estimación del componente estacional en el modelo $Y_{t}=\mu_{t}+\gamma_{t}+\varepsilon_{t}$, con $\mu_{t}=\mu_{t-1}+\eta_{t}$. También se probó a obtener la serie ajustada a partir de un modelo sin componente de nivel. En ambos casos, los resultados fueron muy similares, aunque no exactamente iguales, puesto que la estimación de la componente estacional no es insensible ante la presencia o ausencia de nivel estocástico.
} 
$Y_{t}=\mu_{t}+\gamma_{t}+\sum_{j, k} \lambda_{j k} I_{j k}+\varepsilon_{t}$, con $\mu_{t}=\mu_{t-1}+\eta_{t}$ y $\gamma_{\mathrm{t}}=\sum_{\mathrm{j}=1}^{51} \mathrm{z}_{\mathrm{j}, \mathrm{t}} \gamma_{\mathrm{j}}$, siendo $\mathrm{I}_{\mathrm{jk}}$ una variable de intervención definida como en el apartado 3. El estudio de observaciones anómalas conduce finalmente al modelo que se especifica a continuación (modelo 4) ${ }^{36}$ : $Y_{t}=\mu_{t}+\gamma_{t}+\lambda I_{5190}+\varepsilon_{t}$. Los resultados de la estimación se muestran en los cuadros 8 y 9 y en la figura 2.

\section{CUADRO 8}

Estimación de los parámetros del modelo 4

\begin{tabular}{|l|c|c|c|c|}
\hline Componentes & Irregular & Nivel & \multicolumn{2}{|c|}{$\mathrm{I}_{5190}$} \\
\hline Varianza & $2.3847 \mathrm{e}+009$ & $4.2510 \mathrm{e}+010$ & $\lambda$ & $-1.0825 \mathrm{e}+006$ \\
\hline Ratio Señal-Ruido & $(0.0561)$ & $(1.0000)$ & Estadístico t & -6.6278 \\
\hline
\end{tabular}

\section{CUADRO 9}

Test de significación individual de los componentes del modelo 4 en el vector de estado final

\begin{tabular}{|l|r|l|r|l|c|l|r|}
\hline Componente & \multicolumn{1}{c|}{$\mathrm{t}$} & Componente & $\mathrm{t}$ & Componente & $\mathrm{t}$ & Componente & $\mathrm{t}$ \\
\hline Nivel & 6.8021 & Semana 39 & -7.3153 & Semana 52 & 9.9723 & Semana 13 & 3.5786 \\
\hline Semana 27 & -7.3304 & Semana 40 & -7.2175 & Semana 1 & 9.5966 & Semana 14 & 2.1378 \\
\hline Semana 28 & -7.3403 & Semana 41 & -6.99 & Semana 2 & 9.2297 & Semana 15 & 2.2389 \\
\hline Semana 29 & -7.3416 & Semana 42 & -6.2912 & Semana 3 & 8.7413 & Semana 16 & $1.1993^{*}$ \\
\hline Semana 30 & -7.3427 & Semana 43 & -4.9889 & Semana 4 & 10.259 & Semana 17 & $0.37207 *$ \\
\hline Semana 31 & -7.3439 & Semana 44 & -2.6615 & Semana 5 & 11.276 & Semana 18 & $-0.89067 *$ \\
\hline Semana 32 & -7.345 & Semana 45 & $0.3164 *$ & Semana 6 & 11.769 & Semana 19 & $-1.3668^{*}$ \\
\hline Semana 33 & -7.3384 & Semana 46 & 2.5488 & Semana 7 & 11.106 & Semana 20 & -3.4312 \\
\hline Semana 34 & -7.332 & Semana 47 & 4.7945 & Semana 8 & 9.9648 & Semana 21 & -5.3974 \\
\hline Semana 35 & -7.3357 & Semana 48 & 6.3813 & Semana 9 & 9.3157 & Semana 22 & -6.3949 \\
\hline Semana 36 & -7.3401 & Semana 49 & 7.8442 & Semana 10 & 7.5722 & Semana23 & -6.9101 \\
\hline Semana 37 & -7.3383 & Semana 50 & 8.6423 & Semana 11 & 6.1285 & Semana 24 & -7.1597 \\
\hline Semana 38 & -7.3375 & Semana 51 & 9.9745 & Semana 12 & 4.8656 & Semana 25 & -7.2679 \\
\hline
\end{tabular}

Nota: Se han señalado con * los parámetros no significativos al $90 \%$ de confianza.

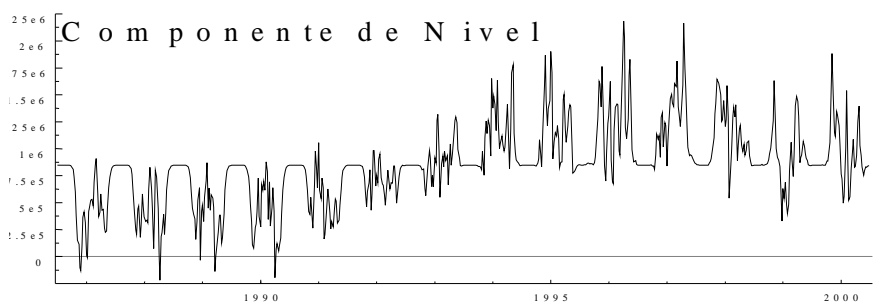

\footnotetext{
${ }^{35}$ Los resultados de los test de significación individual de los componentes del modelo 3 no difieren sustancialmente de los obtenidos para el modelo finalmente propuesto (cuadro 4.5).

36 Aunque se detectaron otras observaciones anómalas, para las especificaciones que incluían un mayor número de variables de intervención no se completó satisfactoriamente el proceso iterativo de estimación.
} 

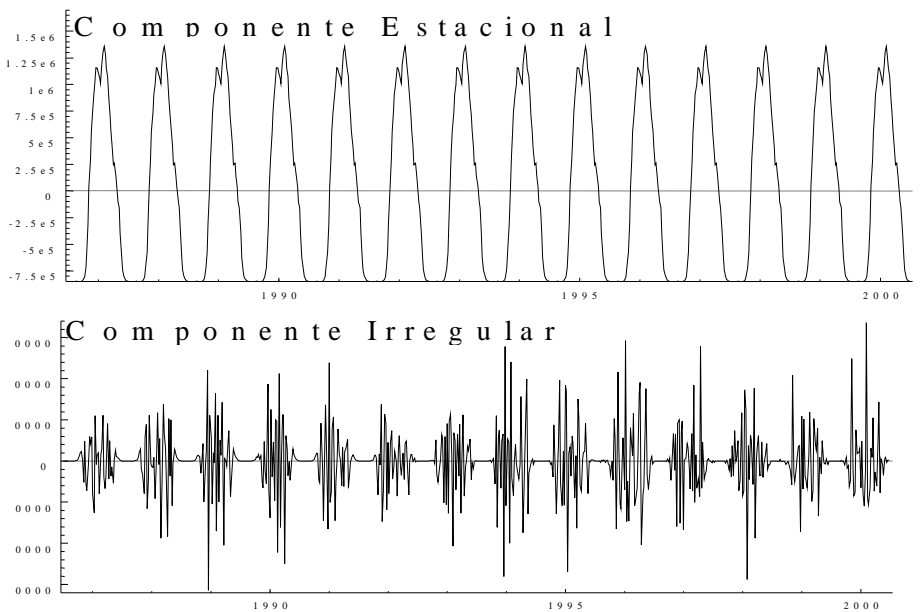

Figura 2. Componentes estimados del modelo 4

\subsection{Examen de la serie filtrada de componentes determinísticos}

La formulación a través de modelos estructurales introduce una especificación más flexible de la inestabilidad presente en el componente tendencial, pero conduce también a la conclusión de la estabilidad del patrón estacional. A efectos comparativos, sería interesante examinar si la especificación de componentes determinísticos cambiantes es una formulación equivalente a la realizada en este apartado. Con este fin, se ha estimado un modelo estructural sobre las tres series resultantes del filtrado de los componentes determinísticos estimados en el apartado 3.1. La representación gráfica de estas series revela un comportamiento estacionario sin presencia alguna de componentes que pudieran expresarse como funciones del tiempo ${ }^{37}$.

A pesar de la claridad de los gráficos, se estimó el modelo estructural básico para las tres series. El proceso posterior de reespecificación permitió excluir la pendiente y el componente estacional. Por tanto, se inició una segunda fase de análisis cuyo objetivo consiste en formular adecuadamente el componente de nivel. En este sentido, se estimó el modelo siguiente (modelo 5): $Y_{t}=\mu_{t}+\varepsilon_{t}$, con $\mu_{t}=\mu_{t-1}+\eta_{t}$. Las estimaciones muestran que las varianzas de los componentes especificados son distintas de cero, lo que nos permite afirmar, para las tres series analizadas, que los valores observados se comportan de manera aleatoria oscilando en torno a un nivel subyacente. Los ratios señal ruido, medidos respecto al componente irregular, fueron $0.0012(0,12 \%), 0.0370(3,7 \%)$ y $0.0325(3,25 \%)$, 
respectivamente. Las estimaciones del nivel en cada momento del tiempo fueron las que se señalan en la figura $3^{38}$.

Al margen de las diferencias observadas para las tres series examinadas, parece interesante comentar que la variabilidad observada en el componente de nivel estocástico es el reflejo de la mayor flexibilidad de los modelos estructurales con respecto a la especificación de cambios estructurales en los componentes determinísticos. Por otra parte, la reducida variabilidad del nivel, aunque estadísticamente significativa, nos llevó a probar con la estimación de un modelo de nivel no estocástico. Los resultados de este segundo modelo indican que, si se asume un nivel fijo, éste es no significativo, resultado totalmente esperado si los componentes determinísticos están correctamente especificados. Es decir, si se optara por no considerar el carácter estocástico del nivel, habría que concluir que el modelo adecuado sólo incorpora componente irregular.
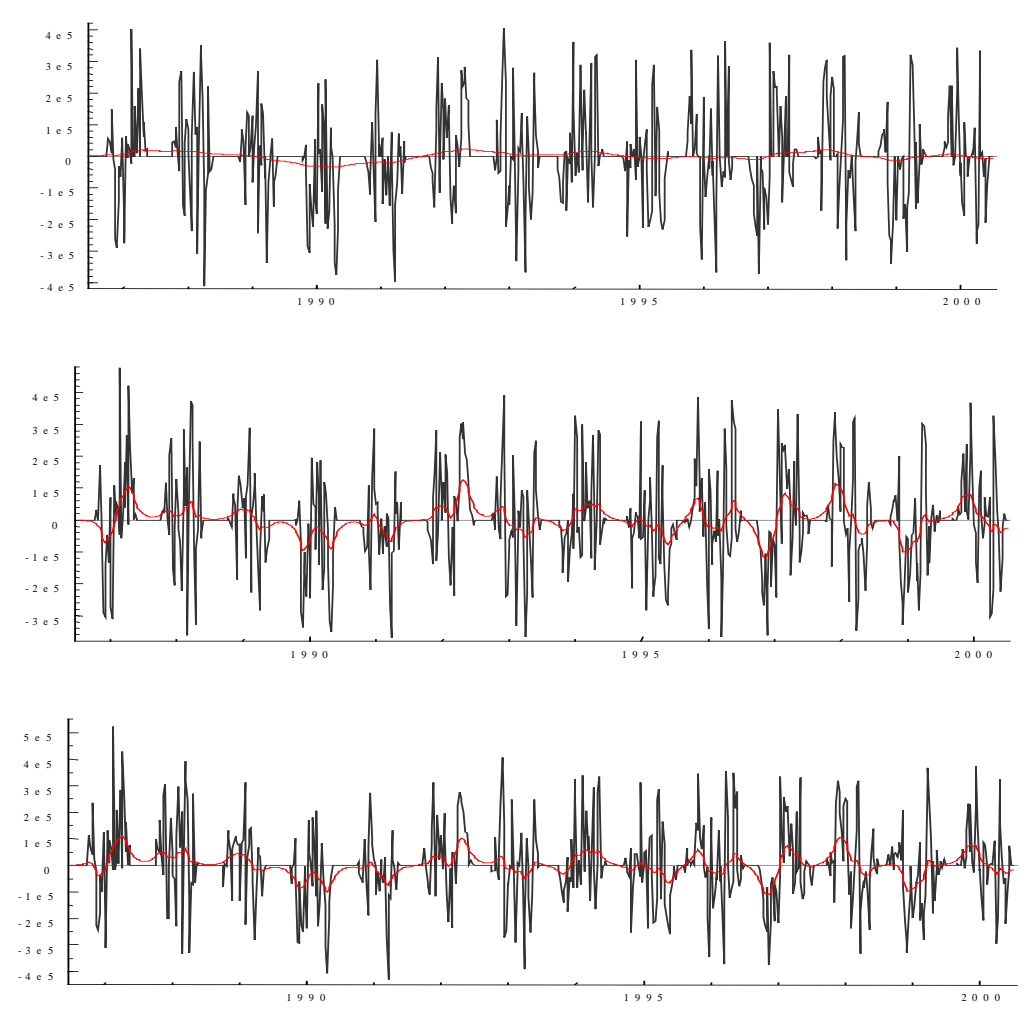

Figura 3. Series $\{F x 1\},\{F x 2\}$ y $\{F x 4\}$ y sus respectivos componentes de nivel

\footnotetext{
${ }^{37}$ Estas representaciones pueden observarse en la figura 3.
} 


\section{Conclusiones}

A partir del estudio de los componentes determinísticos y las raíces unitarias, cabe concluir que la componente estacional de la serie posee una naturaleza determinística dominante alrededor de cambios de nivel y tendencia, también de carácter determinista, en semanas o campañas concretas. Por su parte, el análisis mediante el recurso a modelos estructurales corrobora la estabilidad del patrón estacional de exportación que, sin embargo, se materializa ahora como componente de la serie en torno a un nivel estocástico con raíz unitaria. Se trata, pues, de dos visiones de un mismo fenómeno: la inestabilidad de lo que en el enfoque clásico de series temporales se define como componente tendencial. Una manera de recoger tal inestabilidad consiste en incorporar cambios en los elementos determinísticos; otra es la que admite el modelo estructural como alternativa a un nivel fijo.

Como se ha mostrado en diversos trabajos, si no se incorporan en el modelo cambios estructurales presentes en el comportamiento temporal de una serie, puede concluirse erróneamente que existen tendencias estocásticas asociadas a raíces unitarias. Pero no es sencillo decidir si existen cambios estructurales que deben ser recogidos o si, por el contrario, la respuesta dinámica de la serie a los shocks aleatorios está dirigida por un proceso integrado. En cualquier caso, parece imponerse la conclusión de que, si bien los agentes económicos que protagonizan la actividad productiva y exportadora de tomate canario han reaccionado ante cambios en las reglas de juego del mercado, tal respuesta no se ha traducido en una modificación significativa de la distribución del volumen total exportado en la campaña entre las distintas semanas de la misma.

\section{Bibliografía}

Abeysinghe, T. (1991). «Inappropriate use of seasonal dummies in regression». Economics Letters 36: 175-179.

Abeysinghe, T. (1994). «Deterministic seasonal models and spurious regressions». Journal of Econometrics 61: 259-272.

Cáceres, J.J. (1996). «Contraste de raíces unitarias en datos semanales». Estadística Española 38(141): 139-159.

\footnotetext{
${ }^{38}$ Los modelos antes estimados superaron la fase de diagnóstico.
} 
Cáceres, J.J. (2000). La Exportación de Tomate en Canarias. Elementos para una estrategia competitiva. Ediciones Canarias.

Cáceres, J.J. (2001). «Optimalidad del patrón estacional de las exportaciones canarias de tomate». Estudios de Economía Aplicada 18: 41-66.

Dickey, D.A. y Fuller, W.A. (1981). «Likelihood ratio statistics for autoregressive time series with a unit root». Econometrica 49(4): 1057-1072.

Dickey, D.A. y Pantula, S.G. (1987). «Determining the order of differencing in autoregressive processes». Journal of Business and Economic Statistics 5(4): 455-462.

Durbin, J. y Koopman, S.J. (2001). Time Series Analysis by State Space Methods. Oxford University Press.

Franses, P.H. (1994). Recent advances in modelling seasonality. Econometric Institute Report, 9467/a.

Franses, P.H. (1996). Periodicity and Stochastic Trends in Economic Time Series. Oxford University Press.

Franses, P.H. (1997). «Seasonality in economic time series». En Ullah, A. y Giles, D.E.A. (Eds.) Handbook of Applied Economic Statistics. (Presentado como documento de trabajo del Econometric Institute Report).

Franses, P. H. y Vogelsang, T. (1995). Testing for seasonal unit roots in the presence of changing seasonal means. Econometric Institute. Erasmus University. Rotterdam. Report 9532/A.

Franses, P.H. y Taylor, R. (2000). «Determining the order of differencing in seasonal time series processes». Econometrics Journal 3: 250-264.

Fuller, W. (1976). Introduction to statistical time series. John Wiley \& Sons.

Ghysels, E. (1994). «On the economics and econometrics of seasonality». En Sims, C. (Ed.) Advances in Econometrics. Sixth World Congress, vol. 1, cap. 7.

Ghysels, E., Lee, H.S. y Noh, J. (1994). «Testing for unit roots in seasonal time series». Journal of Econometrics 62: 415-442.

Harvey, A.C. (1989). Forecasting, structural time series models and the Kalman filter. Cambridge University Press.

Harvey, A.C. (1993). Time series models. Harvester Wheatsheaf. 
Harvey, A.C., S.J. Koopman y M. Riani (1995) The modelling and seasonal adjustment of weekly observations, London School of Economics, Discussion Paper EM/95/284.

Hylleberg, S. (1986). Seasonality in regression. Academic Press Inc.

Hylleberg, S. (1992). Modelling seasonality. Oxford University Press.

Hylleberg, S. (1994). «Modelling seasonal variation». En Hargreaves, C.P. (Ed.) Nonstationary time series analysis and cointegration, 153-178. Oxford University Press.

Hylleberg, S., Engle, R.F., Granger, C.W.J. y Yoo, B.S. (1990). «Seasonal integration and cointegration». Journal of Econometrics 44: 215-238.

Kalman, R.E. (1960). «A new approach to linear filtering and prediction problems». Transactions ASME, Series D, Journal of Basic Engineering 82: 35-45.

Kalman, R.E. y Bucy, R.S. (1961). «New results in linear filtering and prediction theory». Transactions ASME, Series D, Journal of Basic Engineering 83: 95-108.

Koopman, S.J. (1992). Diagnostic checking and intradaily effects in time series models. Thesis Publishers. Tinbergen Institute Research Series, 27. Amsterdam.

Koopman, S.J., Harvey, A.C., Doornik, J.A. y Shephard, N. (2000). STAMP: Structural time series analyser, modeller and predictor. Timberlake Consultants.

Mackinnon, J.G. (1991). «Critical values for cointegration tests». En Engle, R.F. y Granger, C.W.J. (Eds.) Long-run economic relationships. Oxford University Press, 267-276.

Miron, J.A. (1994). «The economics of seasonal cycles». En Sims, C. (Ed.) Advances in Econometrics. Sixth World Congress. vol. 1, cap. 6.

Perron, P. y Vogelsang, T.J. (1992a). «Nonstationary and level shifts with an application to purchasing power parity». Journal of Business and Economic Statistics 10: 301-320.

Perron, P. y Vogelsang, T.J. (1992b). «Testing for a unit root in a time series with a changing mean: corrections and extensions». Journal of Business and Economic Statistics 10: $467-470$. 